Gen. Relat. Grav. manuscript No.

(will be inserted by the editor)

\title{
Thermodynamics and Phase transition of the Reissner-Nordström Black Hole surrounded by quintessence
}

\author{
Bouetou Bouetou Thomas • Mahamat Saleh • \\ Timoleon Crepin Kofane
}

Received: date / Accepted: date

\begin{abstract}
We investigate thermodynamics and Phase transition of the Reissner-Nordström black hole surrounded by quintessence. Using thermodynamical laws of black holes, we derive the expressions of some thermodynamics quantities for the Reissner-Nordström black hole surrounded by quintessence. The variations of the temperature and heat capacity with the entropy were plotted for different values of the state parameter related to the quintessence, $\omega_{q}$, and the normalization constant related to the density of quintessence $c$. We show that when varying the entropy of the black hole a phase transition is observed in the black hole. Moreover, when increasing the density of quintessence, the transition point is shifted to lower entropy and the temperature of the black hole decreases.
\end{abstract}

Keywords Black hole - Thermodynamics · Phase transition · Quintessence

\section{Introduction}

Hawking's discovery of thermal radiation from black holes was a complete surprise to most specialists, even though quite a few indications of a close relationship between black hole physics and thermodynamics had emerged before this discovery [1. Recently, a new idea that gravity can be explained as an entropic force caused by the information changes when a material body moves away from the holographic screen was pointed out by Verlinde 2]. He showed that through the holographic principle and the equipartition law of energy that Newton's law of gravitation can arise naturally and unavoidably in

Bouetou B T · Mahamat Saleh · T C Kofane

Department of Physics, Faculty of Science, University of Yaounde I, P.O. Box. 812, Cameroon

Bouetou B T

Ecole Nationale Supérieure Polytechnique, University of Yaounde I, P.O. Box. 8390, Cameroon

Mahamat Saleh

Department of Physics, Higher Teachers' Training College, University of Maroua, P.O. Box.

55, Cameroon,

E-mail: mahsaleh2000@yahoo.fr 
theory in which space is emergent through a holographic scenario, and a relativistic generalization leads to the Einstein equation.

One of the most known solutions of Einstein equation is black hole solution. Black holes are the most fascinating objects in general relativity. One of important characteristics of a black hole is its thermodynamical properties. The discovery that black holes laws are thermodynamical in nature [3, 4, implies that there should be an underlying statistical description of them in terms of some microscopic states. Black hole thermodynamics is widely studied in the literature $5-13$. It is well known that the heat capacity of the Schwarzschild black hole is always negative and so the black hole is thermodynamically unstable. But for the Reissner-Nordström black hole, the heat capacity is negative in some parameter region and positive in other region. Davies pointed out that the phase transition appears in black hole thermodynamics and the second order thermodynamic phase transition takes place at the point where the heat capacity diverges [14 16. Husain and Mann [17] suggest that the specific heat of a black hole becomes positive after a phase transition near the Planck scale. Recently, Jing and Pan [5] investigated second order thermodynamic phase transition for ReissnerNordström black hole. Banerjee, Ghosh and Roychowdhury [18] investigated phase transition in Reissner-Nordström-AdS black hole and its thermodynamics geometry.

From the recent measurements, we can see that our universe is dominated by a mysterious form of energy called "Dark Energy". This kind of energy is responsible of the accelerated expansion of our universe. Today inflation is quite well understood in terms of its phenomenology, but it still has a number of unresolved foundational questions. Despite these, it is certainly clear that cosmic inflation requires a period of cosmic acceleration that cannot be described by a cosmological constant [19, 20. Cosmic inflation is understood to be driven by some matter field typically called the "inflaton" which exhibits an equation of state $p=\rho_{q} \omega_{q}$. In this category we can find quintessence 21, 22, phantom [23, k-essence [24] and quintom [25,26] models.

In this paper, the thermodynamics and phase transition are investigated for the Reissner-Nordström black hole surrounded by quintessence. We consider the metric of a Reissner-Nordström black hole surrounded by quintessence [11,27,29] and, from the laws of black holes thermodynamics, we derived the expressions of thermodynamics quantities of the black hole. Then we plot the behavior of heat capacity and temperature versus the entropy for different values of the density of quintessence in order to extract the effect of the quintessence on the thermodynamical behavior of the black hole.

\section{Thermodynamics phase transition of the black hole}

From Kiselev's investigations 21] on spherically symmetric solutions for Einstein equations describing black holes surrounded by quintessence with the energy momentum tensor, which satisfies the conditions of additivity and linearity, the metric of the Reissner-Nordström black hole space-time surrounded by quintessence can be written as:

$$
d s^{2}=f(r) d t^{2}-f(r)^{-1} d r^{2}-r^{2}\left(d \theta^{2}+\sin ^{2} \theta d \varphi^{2}\right),
$$

where

$$
f(r)=1-\frac{2 M}{r}+\frac{Q^{2}}{r^{2}}-\frac{c}{r^{3 \omega_{q}+1}},
$$


$M$ is the black hole mass, $Q$ is the charge of the black hole, $\omega_{q}$ the quintessential state parameter, $c$ is the normalization factor related to the density of quintessence $\rho_{q}=-\frac{c}{2} \frac{3 \omega_{q}}{r^{3\left(\omega_{q}+1\right)}}$.

The event horizon of the black hole can be found from the following equation

$$
f(r)=0 .
$$

This equation will lead us to two event horizons: the outer and inner horizons given by [11,30]

$$
\begin{aligned}
& r_{+} \simeq M+\sqrt{M^{2}-Q^{2}}+\frac{c\left(M+\sqrt{M^{2}-Q^{2}}\right)^{1-3 \omega_{q}}}{2 \sqrt{M^{2}-Q^{2}}}, \\
& r_{-} \simeq M-\sqrt{M^{2}-Q^{2}}-\frac{c\left(M-\sqrt{M^{2}-Q^{2}}\right)^{1-3 \omega_{q}}}{2 \sqrt{M^{2}-Q^{2}}},
\end{aligned}
$$

respectively.

Taking into account the above equations, the black hole's mass can be expressed as:

$$
M=\frac{1}{2}\left[r_{+}+\frac{Q^{2}}{r_{+}}-\frac{c}{r_{+}^{3 \omega_{q}}}\right] .
$$

The entropy of the black hole is given by the area law as:

$$
S=\frac{A}{4}=\pi r_{+}^{2} .
$$

Substituting (4) into (3), the black hole mass can be rewritten as a function of the black hole entropy

$$
M=\frac{1}{2}\left[\sqrt{\frac{S}{\pi}}+Q^{2} \sqrt{\frac{\pi}{S}}-c\left(\frac{\pi}{S}\right)^{\frac{3 \omega_{q}}{2}}\right] .
$$

With the same considerations, the density of quintessence at the event horizon of the black hole can be expressed in function of the entropy as:

$$
\rho_{q}=-\frac{3 c \omega_{q}}{2}\left(\frac{\pi}{S}\right)^{\frac{3 \omega_{q}+3}{2}}
$$

Its behavior is plotted in Figs. 1 and 2

Using the first law of black hole thermodynamics for the Reissner-Nordström black hole [1]

$$
\delta M=T \delta S+\phi \delta Q,
$$

it is possible to define the other thermodynamics quantities. These quantities are given by:

$$
\begin{aligned}
T & =\left(\frac{\partial M}{\partial S}\right)_{Q}=\frac{1}{4(\pi S)^{\frac{3}{2}}}\left(-\pi^{2} Q^{2}+\pi S+3 c \omega_{q} \pi^{\frac{3 \omega_{q}+3}{2}} S^{\frac{1-3 \omega_{q}}{2}}\right) \\
\phi & =\left(\frac{\partial M}{\partial Q}\right)_{S}=Q \sqrt{\frac{\pi}{S}} \\
C_{\phi} & =T\left(\frac{\partial S}{\partial T}\right)_{\phi}=2 S \frac{-\pi Q^{2}+S+3 c \omega_{q} \pi\left(\frac{\pi}{S}\right)^{\frac{3 \omega_{q}-1}{2}}}{\pi Q^{2}-S-6 c \omega_{q} \pi\left(\frac{\pi}{S}\right)^{\frac{3 \omega_{q}-1}{2}}} \\
C_{Q} & =T\left(\frac{\partial S}{\partial T}\right)_{Q}=-2 S \frac{S-\pi Q^{2}+3 c \omega_{q} \pi^{\frac{1+3 \omega_{q}}{2}} S^{\frac{1-3 \omega_{q}}{2}}}{S-3 \pi Q^{2}+3 c\left(2+3 \omega_{q}\right) \pi^{\frac{1+3 \omega_{q}}{2}} S \frac{1-3 \omega_{q}}{2}}
\end{aligned}
$$




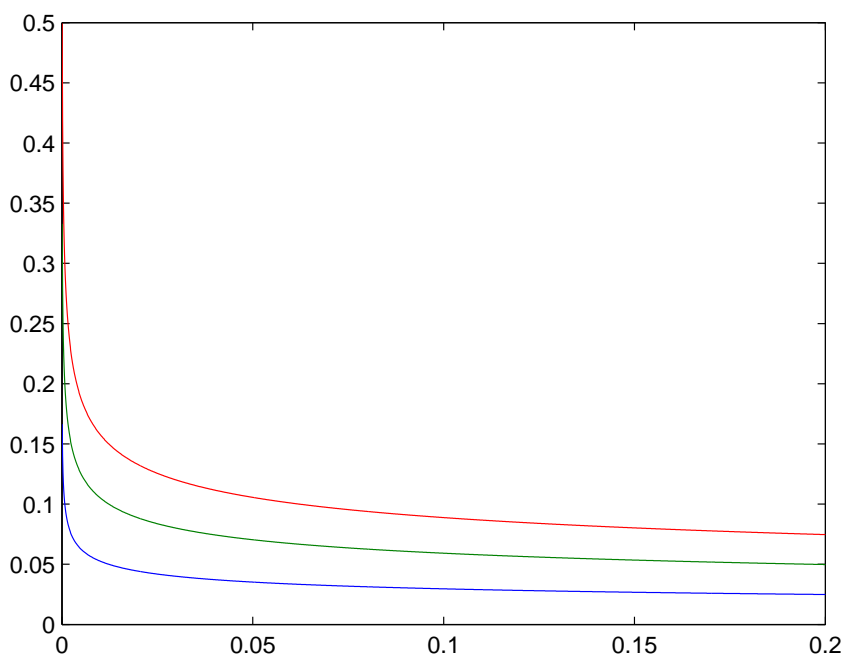

Fig. 1 Variation of the density of quintessence versus entropy for different values of $c$

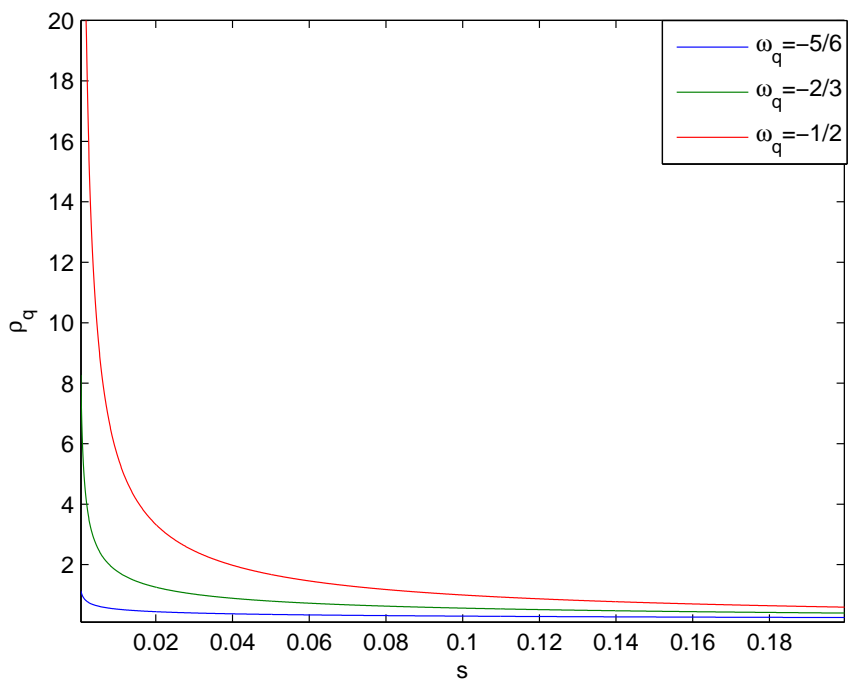

Fig. 2 Variation of the density of quintessence versus entropy for different values of $\omega_{q}$

where $\phi$ represents the potential difference between the horizon and infinity, $T$ is the Hawking temperature of the black hole, $C_{\phi}$ is the heat capacity at constant potential of the black hole and $C_{Q}$ is the heat capacity at constant charge of the black hole. 
In the absence of quintessence $\left(c \omega_{q}=0\right)$, the heat capacity transforms to

$$
C_{\phi}=-2 S<0 \text {. }
$$

This expression is identic to that of the Schwarzschild black hole. In this situation, the only difference between the heat capacities of Schwarzschild and Reissner-Nordström black holes come from the expression of the entropy which depends on the black hole parameters (mass and charge). It means that the Reissner-Nordström black hole is also thermodynamically unstable in the absence of quintessence although there is an infinite discontinuity in the heat capacity at constant charge $C_{Q}$. In fact, Hut 31 argued that although the finite discontinuity in $C_{Q}$ can be classified as a first order phase transition, the infinite discontinuity cannot be considered as a phase change at all. But this infinite discontinuity has a physical significance which in a way transcends that of phase transition. Although this does not affect the internal state of the system as in the case of a phase transition, it indicates transition from a region where only a microcanonical ensemble is appropriate to a region where a canonical ensemble too can be used to describe the system. Now on the phase transition can be observed through the behavior of $C_{\phi}[18$.

Explicitly, we plot the variation of the heat capacity $C_{\phi}$ versus the entropy for different values of the state parameter of the quintessence $\omega_{q}$ and the normalization constant $c$, respectively. Its behavior is represented in Fig. 3 and Fig. 4 , respectively.

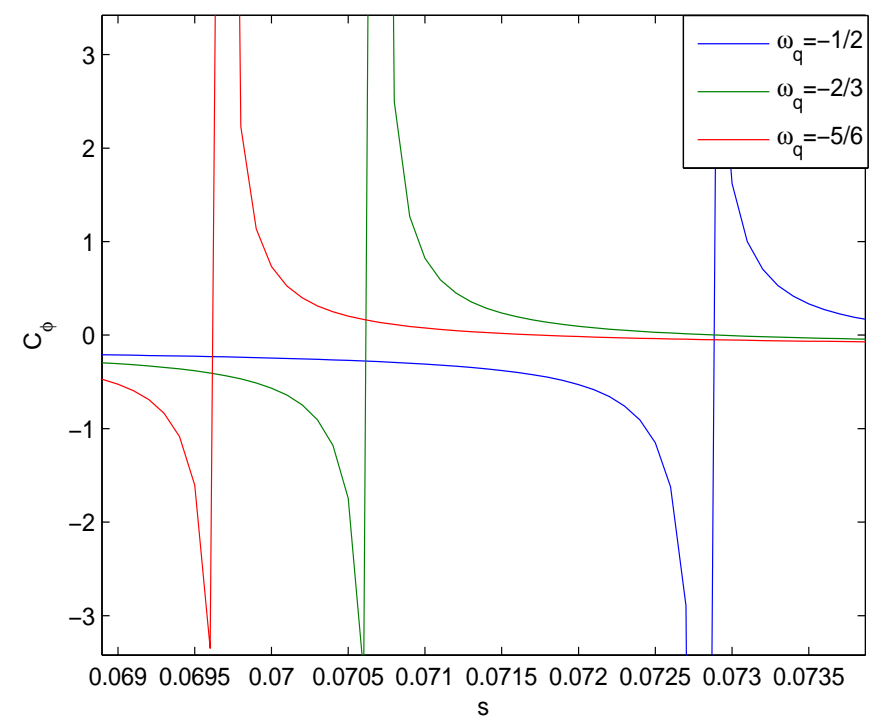

Fig. 3 Variation of the heat capacity at constant potential versus entropy for different values of the state parameter $\omega_{q}$.

Through these figures, we can remark that when varying the entropy, the heat capacity passes a discontinuity point representing the phase transition point of the black hole. Moreover, this point is shifted to lower entropy when decreasing $c$ or $\omega_{q}$. 


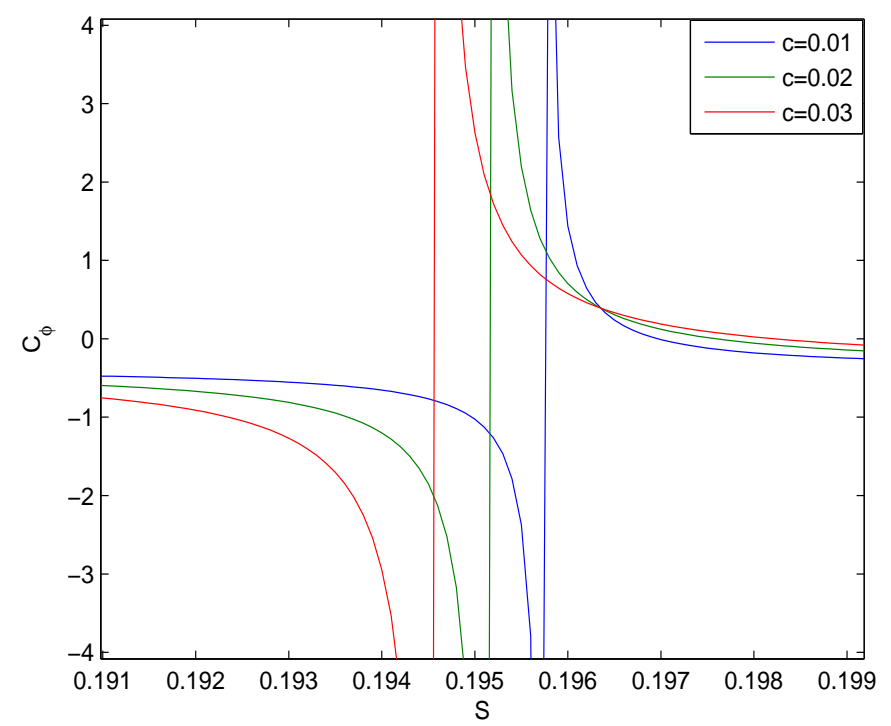

Fig. 4 Variation of the heat capacity at constant potential versus entropy for different values of $\mathrm{c}$.

We also plot the variation of the heat capacity at constant charge $C_{Q}$ versus the entropy for different values of the state parameter of the quintessence $\omega_{q}$ and the normalization constant $c$, respectively. Its behavior is represented in Fig. 5 and Fig. 6 , respectively.

As for the heat capacity at constant potential $C_{\phi}$, we can remark through these figures that when varying the entropy, the heat capacity at constant charge passes a discontinuity point representing the transition from a region where only a microcanonical ensemble is appropriate to a region where a canonical ensemble too can be used to describe the black hole. Moreover, this point is also shifted to lower entropy when decreasing $c$ or $\omega_{q}$.

Comparatively, we can remark that for a given black hole, the discontinuity point of $C_{\phi}$ occurs before that of $C_{Q}$ when increasing the entropy of the black hole. We can also remark that $C_{Q}$ is slightly greater than $C_{\phi}\left(C_{\phi} / C_{Q} \simeq 1.4 \times 10^{-4}\right)$.

Let us now focus on the temperature. To see its behavior, we plot its variation versus the entropy for different values of the state parameter of quintessence, $\omega_{q}$, and the normalization constant, $c$, respectively.

From these figures, we can see that there is no discontinuity of temperature when varying the entropy $S$. We can also remark that the temperature decreases when increasing $c$ or $\omega_{q}$.

\section{Summary and Conclusion}

Quintessence surrounding the Reissner-Nordström black hole modifies the background metric of the black hole. This modification is pointed out by the last term of Eq2 


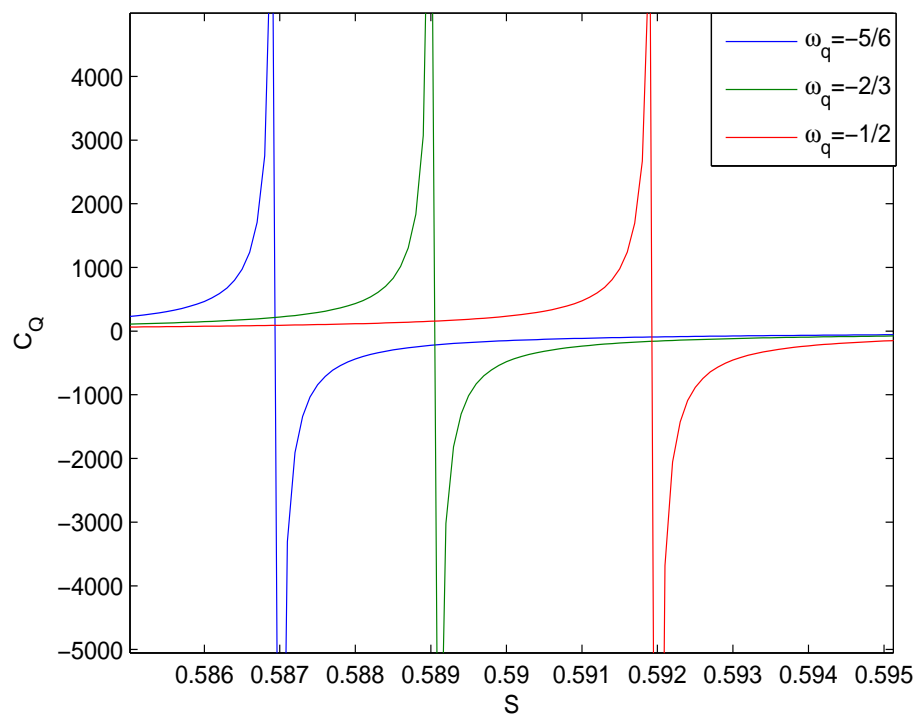

Fig. 5 Variation of the heat capacity at constant charge versus entropy for different values of the state parameter $\omega_{q}$.

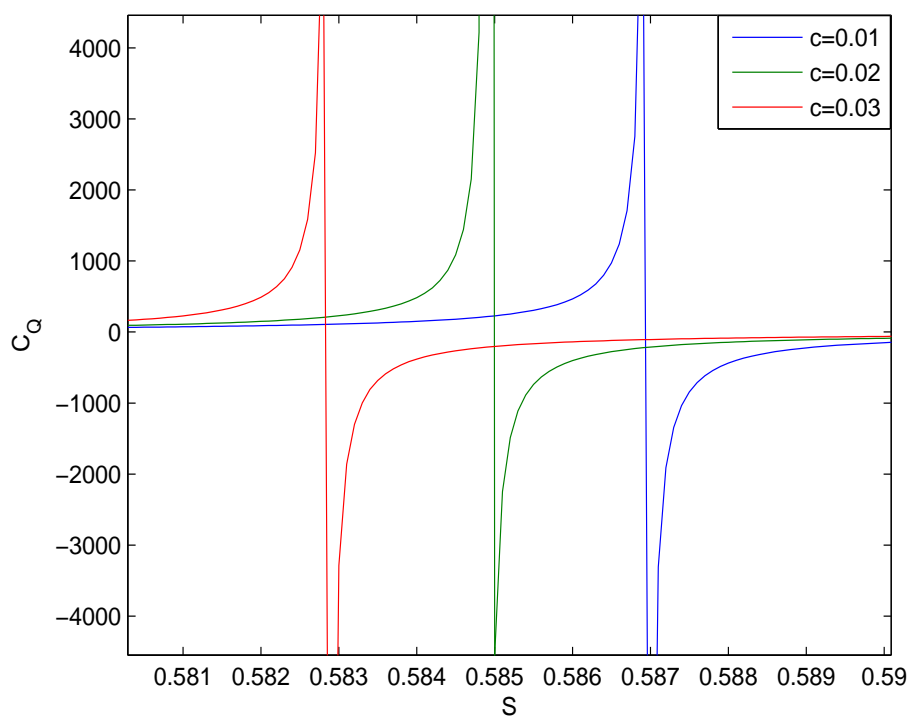

Fig. 6 Variation of the heat capacity at constant charge versus entropy for different values of c. 


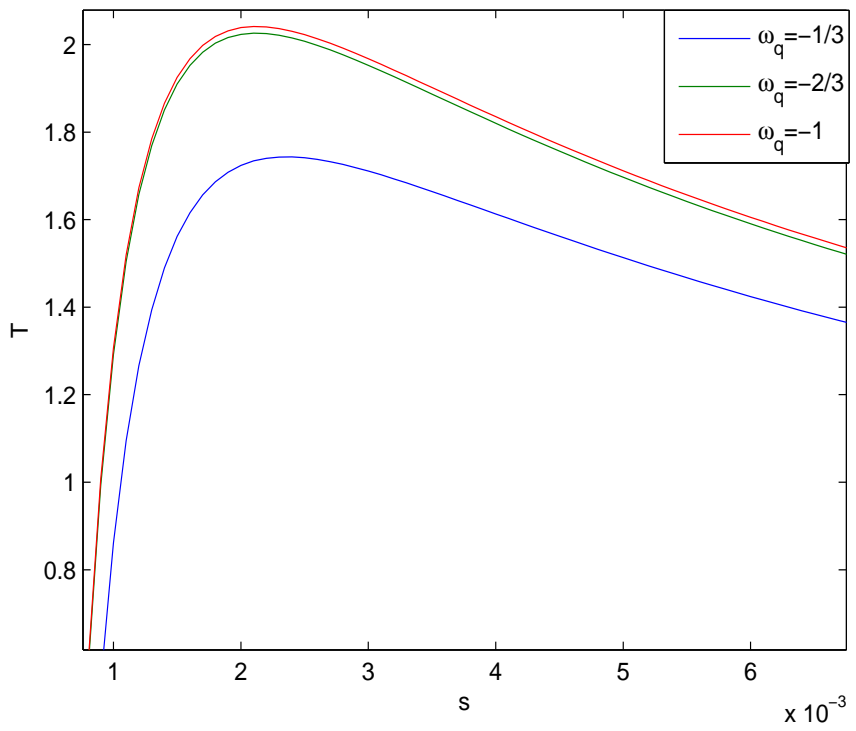

Fig. 7 Variation of the temperature versus entropy for different values of the state parameter $\omega_{q}$.

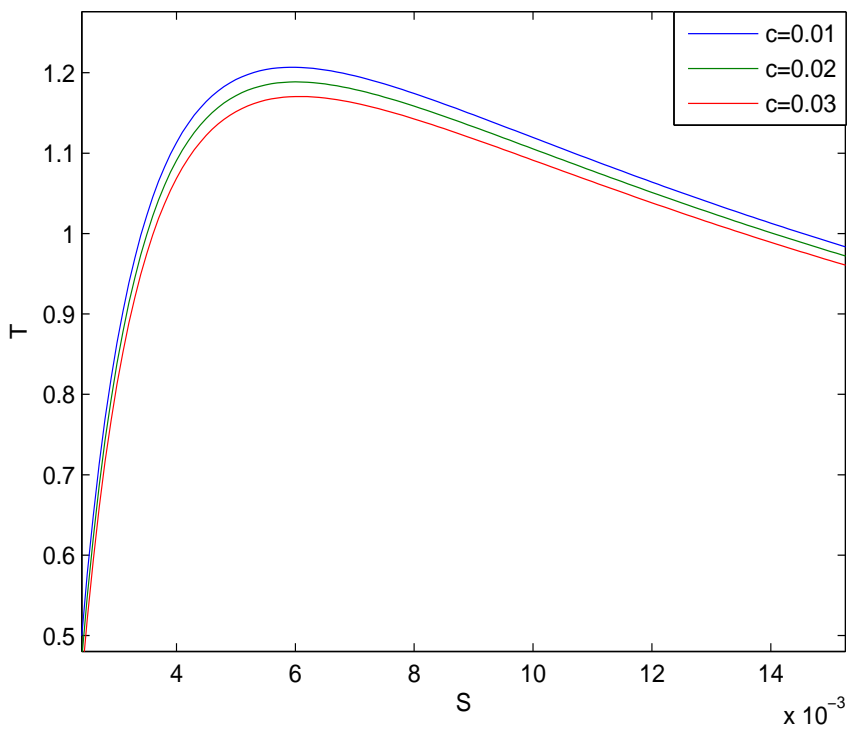

Fig. 8 Variation of the temperature versus entropy for different values of c.

This term is just a perturbation term since the normalization constant related to the 
density of quintessence is actually more smaller than 0.001 32. From the figures plotted above, we can see that the phase transition of the black hole is shifted to lower entropy when increasing $c$ or $\omega_{q}$. Moreover, the temperature of the black hole is decreasing when increasing $c$ or $\omega_{q}$. From Figs. 1 and 2 we can see that in this scale of entropy, increasing $c$ or $\omega_{q}$ means increasing the density of quintessence. Thus we can conclude that when increasing the density of quintessence surrounding the Reissner-Nordström black hole, the phase transition point of the black hole is shifted to lower entropy. We can also conclude that quintessence acts as a cold field decreasing the temperature of the black hole as stepped previously [11. Moreover, we remark that when the density of quintessence vanishes, the black hole becomes thermodynamically unstable and there is no phase transition observed since the heat capacity becomes continuously negative. We can conclude that quintessence helps stabilizing thermodynamically the ReissnerNordström black hole. From Fig[7 we can see that the variation of the temperature becomes more and more negligible when approaching the phantom limit $\left(\omega_{q}=-1\right)$. It will be then interesting to investigate thermodynamical behavior of the ReissnerNordström black hole in the presence of quintom dark energy in future works.

\section{References}

1. V. P. Frolov and I. D. Novikov, Black Hole Physics: Basic concepts and new developments, Kluwer (1997) chap. 12

2. E. Verlinde, JHEP 1104 (2011) 029.

3. S. W. Hawking, Phys. Rev. D 13 (1976) 191

4. S. W. Hawking and Don N. Page, Commun. Math. Phys. 87 (1983) 577.

5. J. Jing and Q. Pan, Phys. Lett. B 660 (2008) 13.

6. P. Qiyuan and J. Jing, Phys. Rev. D 78 (2008) 065015.

7. G. Amit and P. Mitra, Phys. Lett. B 357 (1995) 295.

8. J. Qing-Quan and W. Shuang-Qing, Phys. Lett. B 635 (2006) 151.

9. A.S.D. Gonçalo, Phys. Rev. D 79 (2009) 044013.

10. P. Yu and N.E. Firsova, Nucl. Phys. B 486 (1997) 371.

11. S. Mahamat, T.B. Bouetou and T.C. Kofane, Commun. Theor. Phys. 55 (2011) 291.

12. G. Q. Li, Pis'ma v ZhETF, 86 (2007) 179.

13. S. Chen, B. Wang and R. Su, Phys. Rev. D 77 (2008) 124011.

14. P.C.W. Davies, Proc. Roy. Soc. London A 353(1977)499.

15. P.C.W. Davies, Rep. Prog. Phys. 41(1978)1313.

16. P.C.W. Davies, Class. Quantum Grav. 6(1989)1909.

17. V. Husain and R. B. Mann, Class. Quantum Grav. 26 (2009) 075010

18. R. Banerjee, S. Ghosh and D. Roychowdhury, Phys. Lett. B 696 (2011) 156162.

19. C. Rolando, G. Tame, L. Yoelsy, M. Osmel and Q. Israel, Phys. Rev. D 67 (2003) 083501.

20. C. Liu, The Handy Astronomy Answer Book, (Visible Ink Press, Malaysia, 1968) p 51.

21. V.V. Kiselev, Class. Quantum Grav. 20 (2003) 1187.

22. Z. Shuang-Yong, Phys. Lett. B 660 (2008) 7.

23. K. Martin and S. Domenico, Phys. Rev. D 74 (2006) 123503.

24. R. YANG and X. GAO, Chin. Phys. Lett. 26 (2009) 089501.

25. Z.-K. Guo, Y.-S. Piao, X. Zhang and Y.-Z. Zhang, Phys. Lett. B 608 (2005) 177.

26. J.-Q. Xia, B. Feng and X. Zhang, Phys. Rev. D 74 (2006) 123503.

27. N. Varghese and V.C. Kuriakose, Gen. Relativ. Gravit. 41 (2009) 1249.

28. C.-Y. WANG, Yu ZHANG, Y.-X. GUI and J.-B. LÜ, Commun. Theor. Phys. 53 (2010) 882 .

29. S. Mahamat, T.B. Bouetou and T.C. Kofane, Chin. Phys. Lett. 26 (2009) 109802.

30. S. Mahamat, T. B. Bouetou and T. C. Kofane, Astrophys. Space Sci. 333 (2011) 449.

31. P. Hut, Mon. Not. R. Astr. Soc. 180 (1977) 379.

32. Yu Zhang and Y.X. Gui, Class. Quantum Grav. 23 (2006) 6141. 